\title{
Effect of Direct-Current Biasing on the Dielectric Properties of Barium Strontium Titanate
}

\author{
Jih-Wei Liou and Bi-Shiou Chiou* \\ Department of Electronics Engineering and Institute of Electronics, National Chiao Tung University, \\ Hsinchu, Taiwan
}

\begin{abstract}
$\mathrm{Ba}_{1-x} \mathrm{Sr}_{x} \mathrm{TiO}_{3}(\mathrm{BST}, x=0$ to $x=1)$ ferroelectric ceramics doped with $1.0 \mathrm{~mol} \% \mathrm{MgO}$ and $0.05 \mathrm{~mol} \% \mathrm{MnO}_{2}$ were prepared with a rate-controlled sintering profile. As the strontium molar fraction $x$ increased, the average grain size decreased from $14 \mu \mathrm{m}$ for $x=0$ to $2 \mu \mathrm{m}$ for $x=1$. Temperature dependence of the dielectric properties was measured as a function of dc biasing. At $10^{\circ} \mathrm{C}$ above the Curie temperature, the changes of dielectric constant at $5000 \mathrm{~V} /$ cm were $28 \%, 17 \%, 26 \%$, and $36 \%$ for $x=0,0.25,0.5$, and 0.75 , respectively. The reduction of dielectric constant by the applied dc biasing was fitted by a phenomenological equation that was based on Devonshire's theory. From this phenomenological equation, an anharmonic coefficient, which was an order parameter of the anharmonic interactions, was calculated for each composition. As the strontium molar fraction increased, these coefficients increased from $2.45 \times 10^{-19} \mathrm{~cm}^{2} \cdot V^{-2}$ for $x=0$ to $5.90 \times 10^{-19} \mathrm{~cm}^{2} \cdot \mathrm{V}^{-2}$ for $x=0.75$. A similar trend was observed on the de field dependence of the dielectric loss, except at high field for $x=0$, of which a high loss was obtained. A two-element circuit model was proposed to explain the effect of high dc biasing on the dielectric loss.
\end{abstract}

\section{Introduction}

$\mathrm{F}$ ERROELECTRIC barium strontium titanate (BST, $\mathrm{Ba}_{1-x} \mathrm{Sr}_{x} \mathrm{TiO}_{3}$ ) is of great interest for many practical applications. Many studies have been done on the temperature-dependent dielectric properties of BST. ${ }^{1-5}$ The effects of various dopants on the dielectric properties of BST also have been investigated extensively. ${ }^{6-9}$ However, there are few studies on the dielectric constant as a function of the dc-biasing field. Previous works have suggested that BST can be applied as a microwave phase shifter ${ }^{10,11}$ and tunable capacitor. ${ }^{12-14}$ A BST system must exhibit the following characteristics if it is to be applied as an electrically tunable capacitor: a variation of dielectric constant under dc biasing greater than $\sim 30 \%$ at the working temperature; low dielectric loss under dc biasing; and insensitivity of dielectric properties to ambient temperature variation. Accurate measurement of these dielectric properties provides scientists and engineers with valuable information to properly incorporate BST materials into their intended applications for many solid device designs. The purpose of this study is to understand the dielectric behavior of BST under dc biasing and discuss the important factors that influence the dielectric characteristics of BST.

W. Huebner-contributing editor

Manuscript No. 191621. Received August 6, 1996; approved April 7, 1997.

Supported by Chung-Shan Institute of Science and Technology under Contract No. CS-85-0210-D-009-009 and supported in part by National Science Council of Taiwan, ROC, under Contract No. NSC 85-2216-E-009-012.

Presented at the 98th Annual Meeting of the American Ceramic Society, Indianapolis, IN, April 15, 1996 (Electronics Division, Paper No. E-26-96).

${ }^{*}$ Member, American Ceramic Society.
In this study, the BST system was doped with $\mathrm{MnO}_{2}$ and $\mathrm{MgO}$, and it was prepared for the investigation of dielectric behavior under the dc-biasing field at various temperatures. The strontium molar fraction $x$ ranged from 0 to 1 at intervals of 0.25 to vary the Curie temperature $\left(T_{\mathrm{C}}\right)$ of the BST system. Dopant $\mathrm{MnO}_{2}$ was used to trap the electrons to obtain low-loss dielectrics, ${ }^{8}$ whereas dopant $\mathrm{MgO}$ was used as a grain-growth inhibitor. ${ }^{6}$ A nonisothermal rate-controlled firing profile was used to obtain a uniform sample microstructure. ${ }^{6}$

\section{Experimental Procedure}

Doped $\mathrm{Ba}_{1-x} \mathrm{Sr}_{x} \mathrm{TiO}_{3}(x=0,0.25,0.5,0.75$, and 1) polycrystalline samples were prepared. Commercial $\mathrm{BaCO}_{3}$, $\mathrm{SrCO}_{3}, \mathrm{TiO}_{2}, \mathrm{MnO}_{2}$, and $\mathrm{MgO}$ powders (Merck and Co., Inc., Darmstadt, German) were ball milled with acetone and alumina balls for $24 \mathrm{~h}$. Excess $1.0 \mathrm{~mol}_{\%} \mathrm{TiO}_{2}$ was added to obtain a $\mathrm{TiO}_{2}$-rich liquid phase during sintering. ${ }^{15} \mathrm{In}$ all the samples, the amount of $\mathrm{MgO}$ and $\mathrm{MnO}_{2}$ additives were 1.0 and 0.05 mol\%, respectively. After the mixture was dried by an IR lamp, it was calcined in an alumina crucible at $1100^{\circ} \mathrm{C}$ for $2 \mathrm{~h}$ in air. The powder then was mixed with a small amount of poly(vinyl acetate) (PVA) binder and pressed to form disk-shaped samples at $180 \mathrm{MPa}$. Sintering of samples was conducted with a controlled firing profile 6 after the binder was burned out at $400^{\circ} \mathrm{C}$ for $3 \mathrm{~h}$. Samples were then heated at $100^{\circ} \mathrm{C} / \mathrm{min}$ to $1200^{\circ} \mathrm{C}$, with a 6 min hold at $1200^{\circ} \mathrm{C}$, and then to the sintering temperature of $1400^{\circ} \mathrm{C}$ at the same heating rate. The sintering profile was conducted in air in a $1700^{\circ} \mathrm{C}$ box furnace with a maximum heating rate of $\sim 120^{\circ} \mathrm{C} / \mathrm{min}$ (Model 51314, Lindberg, Watertown, WI) with a temperature controller (Model 818P, Endotherm Corp., Reston, VA). The sintering time was $18 \mathrm{~min}$ at $1400^{\circ} \mathrm{C}$, and the samples then were cooled to room temperature at a rate of $180^{\circ} \mathrm{C} / \mathrm{h}$. Phase identification of these specimens was conducted by powder X-ray diffractometry (XRD) (Model Dmax-B, Rigaku Co., Tokyo, Japan) with $\mathrm{Cu} K \alpha$ radiation. A thin gold film was deposited on the surface of the as-sintered pellet. Scanning electron microscopy (SEM) (Model S-750, Hitachi, Ltd., Tokyo, Japan) was used to examine the microstructures. The average grain size was determined by the linear-intercept method from the micrograph of the assintered sample.

Pellet-shaped samples, $0.86 \mathrm{~cm}$ in diameter, were polished with $\mathrm{SiC}$ paper (grit 800 ) to $0.4 \mathrm{~mm}$ thickness. Extreme scrutiny was taken in the polishing and in thickness measuring of the sample to assure flat and parallel surfaces. The thickness deviation was within the experimental error of the measurement. Samples were electroded by rubbing In-Ga (40:60) alloy on both surfaces to provide ohmic contacts. The dielectric constant of each sample was calculated from the measured capacitance and the specimen geometry. The capacitance was measured with an impedance-capacitance-resistance (LCR) meter (Model HP4275A, Hewlett-Packard, Palo Alto, CA) at $10 \mathrm{kHz}$. A $200 \mathrm{~V}$ dc power supply was connected to the LCR meter as the external dc bias. The current-voltage characteristics were measured (Model HP4140, Hewlett-Packard). 


\section{Results and Discussion}

\section{(1) X-ray Analysis and Grain-Size Measurements}

Typical XRD patterns of sintered samples (BST doped with $1.0 \mathrm{~mol} \% \mathrm{MgO}$ and $0.05 \mathrm{~mol} \% \mathrm{MnO}_{2}$ ) are shown in Fig. 1. The XRD patterns show that the crystal structures are tetragonal phase for $x=0$ and 0.25 and that the structures are cubic phase for $x=0.5,0.75$, and 1. The labeled Miller index for each diffraction peak is based on the space groups of the two types of crystal structure. The tetragonal space group for $x=$ 0 and 0.25 and the cubic space group for $x=0.5,0.75$, and 1 are, respectively, $P 4 m m$ and $P m 3 m$. Syamaprasad et al. ${ }^{1}$ have reported XRD patterns of the undoped BST system and have revealed that there are two or more phases for $x=0.3$ and 0.5 that are prepared by conventional solid-solution sintering. In the current work, with a rate-controlled sintering profile, ceramics of doped BST without apparent multiphase solution have been obtained. The unit-cell parameters based on the least-squares refinement with more than nine diffraction pattern peaks are calculated and listed in Table I. The unit-cell volume calculated from the lattice constants decreases as the strontium molar fraction increases; this is attributed to the smaller $\mathrm{Sr}^{2+}$ ionic radius compared to the $\mathrm{Ba}^{2+}$ ionic radius. The effect of dopants on the microstructure of the BST system is shown in Fig. 2. As a result of a rate-controlled sintering profile, no abnormal grain growth is observed in Fig. 2. The average grain sizes of all compositions also are listed in Table I. These values range from 14 to $2 \mu \mathrm{m}$ and decrease with an increase in strontium molar fraction. It is argued that the ceramic and liquid phases become more refractory as strontium content increases. Therefore, under the same sintering condition, the grain-growth inhibitor $\mathrm{MgO}$ becomes more difficult to dissolve in the more-refractory samples.

\section{(2) Temperature Dependence of Dielectric Properties}

The temperature dependence of the dielectric constant measured at $10 \mathrm{kHz}$ as a function of dc-biasing field for the doped BST system is shown in Fig. 3. The dielectric constant decreases with increasing dc-biasing field in both ferroelectric

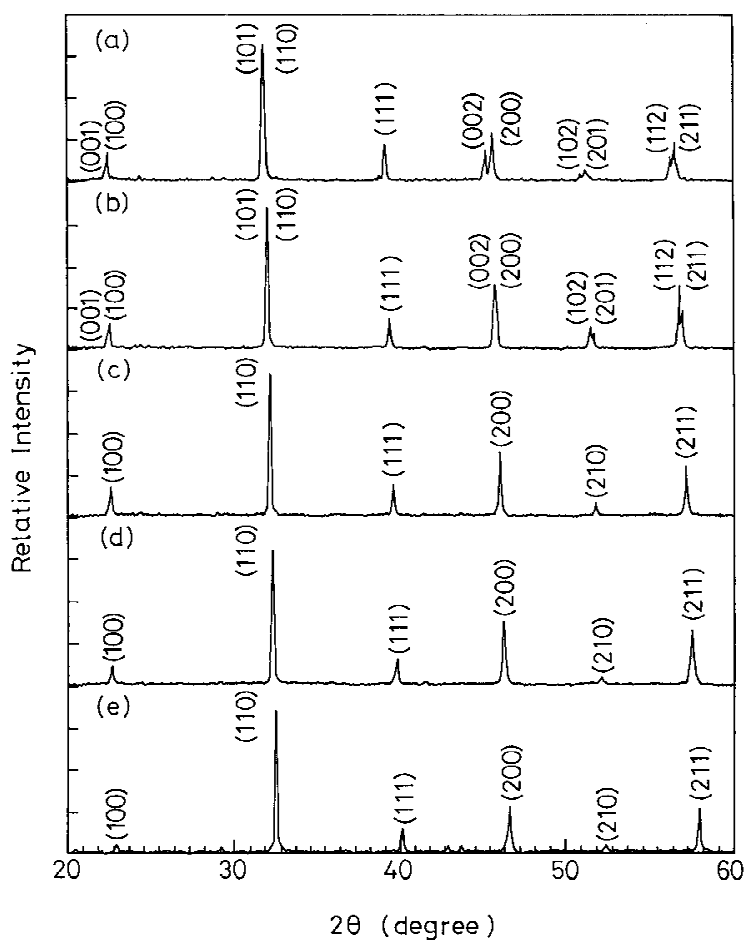

Fig. 1. XRD patterns for BST doped with $1.0 \mathrm{~mol} \% \mathrm{MgO}$ and 0.05 mol\% $\mathrm{MnO}_{2}$ : (a) $x=0$, (b) $x=0.25$, (c) $x=0.5$, (d) $x=0.75$, and (e) $x=1$. and paraelectric states. The Curie temperature is defined as the temperature of maximum dielectric constant. The maximum dielectric constant $\left(k_{\max }\right)$ for the doped BST system ranges from $8 \times 10^{3}$ to $1.2 \times 10^{4}$ for $x \leq 0.75$. The dielectric peak for $x=1$ is beyond the temperature range of the measuring apparatus. The values of dielectric constant are in a reasonable range compared to pure BST reported from other work. ${ }^{16-20}$ Hence, dopants $\mathrm{MgO}$ and $\mathrm{MnO}_{2}$ do not significantly change the ferroelectric feature of the BST system. Figure 3 shows that the higher the strontium molar fraction, the broader the ferroelectric-to-paraelectric transition. In the doped BST system, the half-height width of the Curie peak is estimated to be $\sim 20^{\circ}$ to $40^{\circ} \mathrm{C}$, increasing with strontium molar fraction for $x=0$ to $x=0.75$. There are several causes that may result in transition broadening, such as composition variation and internal stress. Because only single-phase material is observed in the XRD patterns, it is believed that composition variation is not the major cause for transition broadening. It is argued that transition broadening can be attributed to the larger internal stress in the finer grains. ${ }^{19}$ As the grain size decreases, the remaining internal stress due to ferroelectric deformation becomes more difficult to release. The remaining internal stress can vary in value to a larger extent, leading to a larger distribution width. Hence, the Curie peak broadens and the dielectric constant at the ferroelectric state is higher. ${ }^{16-20}$ Deb et al. ${ }^{3}$ reported that $T_{\mathrm{C}}$ of an undoped $\mathrm{BaTiO}_{3}$ was $128^{\circ} \mathrm{C}$. Kinoshita and Yamaji ${ }^{16}$ prepared $\mathrm{BaTiO}_{3}$ of various grain sizes ranging from 1 to 53 $\mu \mathrm{m}$ and found that $T_{\mathrm{C}}$ values were slightly influenced by the grain size and ranged from $120^{\circ}$ to $122^{\circ} \mathrm{C}$. In the current study, $T_{\mathrm{C}}$ for the doped $\mathrm{BaTiO}_{3}$ is $104^{\circ} \mathrm{C}$, which is lower than the reported data. This is due to the addition of $\mathrm{Mn}^{2+}$ ions that occupy the $\mathrm{Ti}^{4+}$ ion sites. The lower valence state of the $\mathrm{Mn}^{2+}$ ion, with the resulting oxygen vacancy leading to a "break" of the cooperative vibration of the Ti-O chains, is responsible for the lower $T_{\mathrm{C}}$ of the manganese-doped system. ${ }^{8}$ The $T_{\mathrm{C}}$ values that decrease with strontium molar fraction also are listed in Table I. The lattice parameters (which are represented by the root of the calculated unit-cell volume from X-ray analysis data) and $T_{\mathrm{C}}$ values of different strontium molar fractions are shown in Fig. 4. A linear relation of lattice parameters with strontium molar fraction is observed. In the perovskite structure, the $\mathrm{Sr}^{2+}$ and $\mathrm{Ba}^{2+}$ ionic radii at sixfold coordinate are 1.16 and $1.36 \AA$, respectively. ${ }^{9}$ Because of the ionic radius difference, the lattice parameter $V_{\mathrm{C}}^{1 / 3}$ decreases with increasing strontium molar fraction. Furthermore, with decreasing lattice parameter, which is the characteristic distance of spontaneous polarization of ferroelectric ceramics, the phase transition occurs at a lower temperature. A linear relation of $T_{\mathrm{C}}$ with molar fraction $x$ occurs for $x \leq 0.75$ as a straight line, as shown in Fig. 4. From this linearity, the $T_{\mathrm{C}}$ of the doped BST system with other strontium molar fractions can be designed by controlling the strontium fraction, and an empirical equation can be obtained for $x \leq 0.75$ :

$$
T_{\mathrm{C}}\left({ }^{\circ} \mathrm{C}\right)=104-288 x
$$

where the $x$ is the strontium molar fraction.

The reported $T_{\mathrm{C}}$ for $x=1$ is lower than the value predicted by Eq. (1). Viana et al. ${ }^{5}$ have reported that the ferroelectric transition of $\mathrm{SrTiO}_{3}$ occurs at $40 \mathrm{~K}$ and that the structural phase transition occurs at $105 \mathrm{~K}$. One possible reason for the reduction of $T_{\mathrm{C}}$ is that the long-range ferroelectric order is suppressed by quantum fluctuation at low temperatures.

\section{(3) Biasing Field Dependence of Dielectric Properties}

Figure 3 also shows that the dielectric constant decreases with increasing bias field $E$. The change of dielectric constant in the ferroelectric state is not apparent for the sample with $x=$ 0 . The domain width motion under dc biasing is believed to be the factor influencing the dielectric behavior. The dc-biasing effect usually is stronger in the paraelectric state than in the ferroelectric state for the sample where $x=0$ in Fig. 3. As for strontium-containing samples, a broad ferroelectric-to-para- 
Table I. Structural Parameters, Curie Temperatures, and Average Grain Sizes for the 1.0-mol\%-MgO- and $0.05-\mathrm{mol} \%-\mathrm{MnO}_{2}$-Doped $\mathrm{Ba}_{1-x} \mathrm{TiO}_{3}$ System

\begin{tabular}{|c|c|c|c|c|c|}
\hline $\begin{array}{l}\text { Strontium } \\
\text { molar } \\
\text { fraction }\end{array}$ & Lattice constants $(\AA)$ & Structure & $\begin{array}{c}\text { Volume of } \\
\text { unit cell }\left(\AA^{3}\right)\end{array}$ & $\begin{array}{c}\text { Average } \\
\text { grain size } \\
(\mu \mathrm{m})\end{array}$ & $\begin{array}{c}\text { Curie } \\
\text { temperature, } \\
T_{\mathrm{C}}\left({ }^{\circ} \mathrm{C}\right)\end{array}$ \\
\hline$x=0$ & $a=3.994, c=4.032$ & Tetragonal & 64.32 & 13.8 & 104 \\
\hline$x=0.25$ & $a=3.980, c=3.992$ & Tetragonal & 63.23 & 9.5 & 34 \\
\hline$x=0.5$ & $a=3.960$ & Cubic & 62.10 & 4.6 & -40 \\
\hline$x=0.75$ & $a=3.931$ & Cubic & 60.74 & 2.8 & -132 \\
\hline$x=1$ & $a=3.905$ & Cubic & 59.55 & 2.2 & $\dagger$ \\
\hline
\end{tabular}
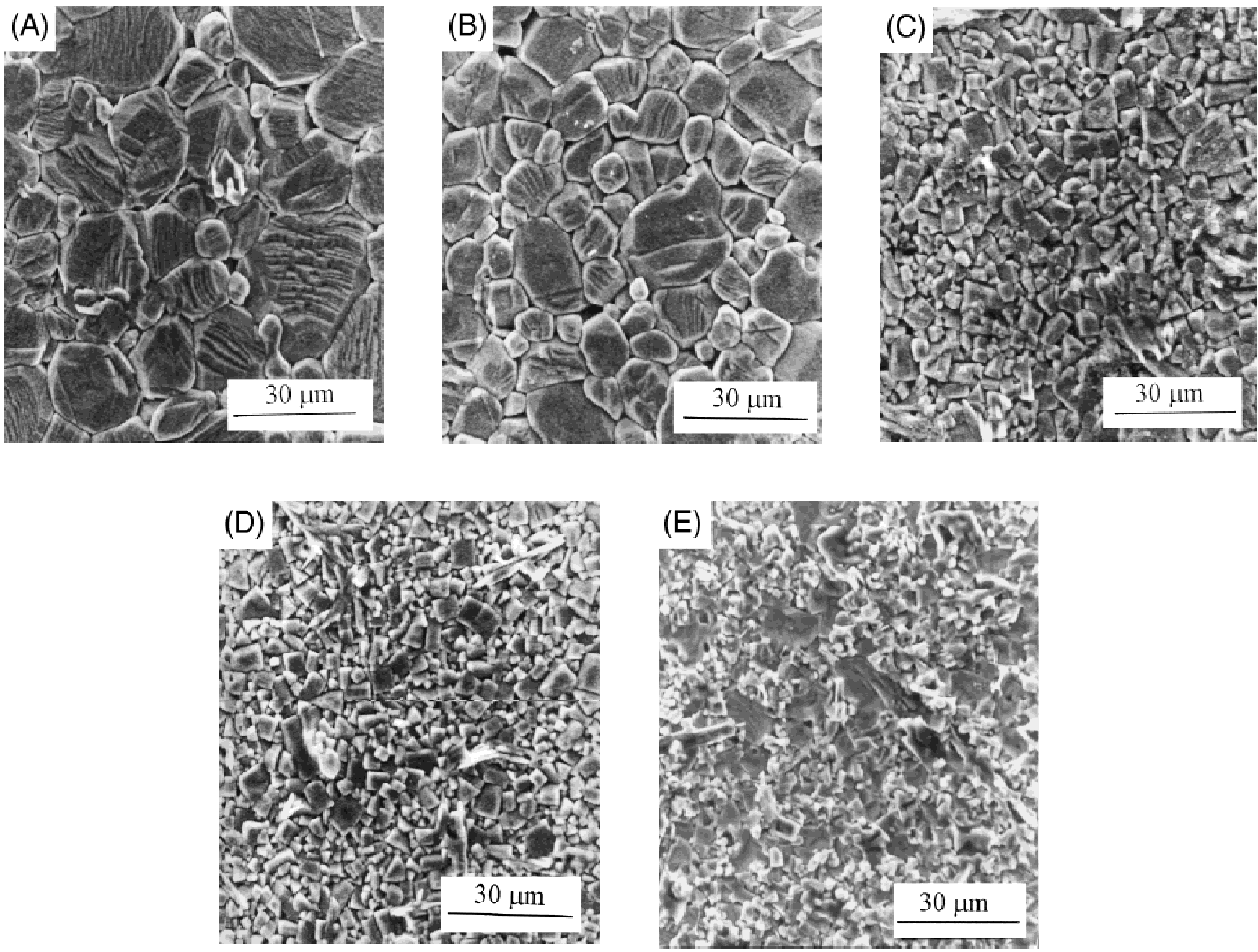

Fig. 2. Microstructures of BST doped with $1.0 \mathrm{~mol} \% \mathrm{MgO}$ and $0.05 \mathrm{~mol} \% \mathrm{MnO}_{2}$ : (A) $x=0$, (B) $x=0.25$, (C) $x=0.5$, (D) $x=0.75$, and (E) $x=1$.

electric transition occurs. Therefore, the dc-biasing effect on the dielectric constant occurs in both the ferroelectric and paraelectric states. Change in dielectric constant increases with increasing strontium content, and the maximum change occurs at $T_{\mathrm{C}}$, as shown in Fig. 3. The dielectric tunability $D_{\mathrm{t}}$ is defined at a specific working temperature $T$ and dc-biasing field $E$ as,

$$
D_{\mathrm{t}}(T, E)=\left(1-\frac{k^{\prime}}{k_{0}^{\prime}}\right) \times 100 \%
$$

where $k_{0}^{\prime}$ and $k^{\prime}$ are the real part of the relative permittivity (i.e., dielectric constant) under zero bias field and under bias field $E$, respectively. As listed in Table II, $D_{\mathrm{t}}$ with $5 \mathrm{kV} / \mathrm{cm}$ biasing field at $T_{\mathrm{C}}$ and $T_{\mathrm{C}}+10^{\circ} \mathrm{C}$ increase with $x$ for $x=0.25$ to $x=0.75$.

According to Devonshire's theory, ${ }^{21}$ in the perovskite cubic structure, titanium ions oscillate in an anharmonic potential of the form $a r^{2}+b r^{4}$, where $r$ is the position of the titanium ion. The Helmholtz free energy $F(P, T)$ of the titanium ion can be expanded in even powers of the polarization $P$ with coefficients that are a function of the temperature only; that is,

$$
F(P, T)=F(0, T)+A(T-\theta) P^{2}+B P^{4}+C P^{6}
$$

where $\theta$ is the Curie-Weiss temperature, and $A, B$, and $C$ the expansion coefficients. In the paraelectric state, the free energy increases as polarization, and there is only one minimum, at polarization $P=0$. If a small field $E$ is considered to apply on the materials, that is, approximately the minimum, the $P^{6}$ term can be neglected. Moreover, in the paraelectric state, where the polarization is much smaller than that in the ferroelectric state, neglecting the $P^{6}$ term in the expansion of free energy is appropriate. The dielectric constant $k^{\prime}$ can be obtained by taking 


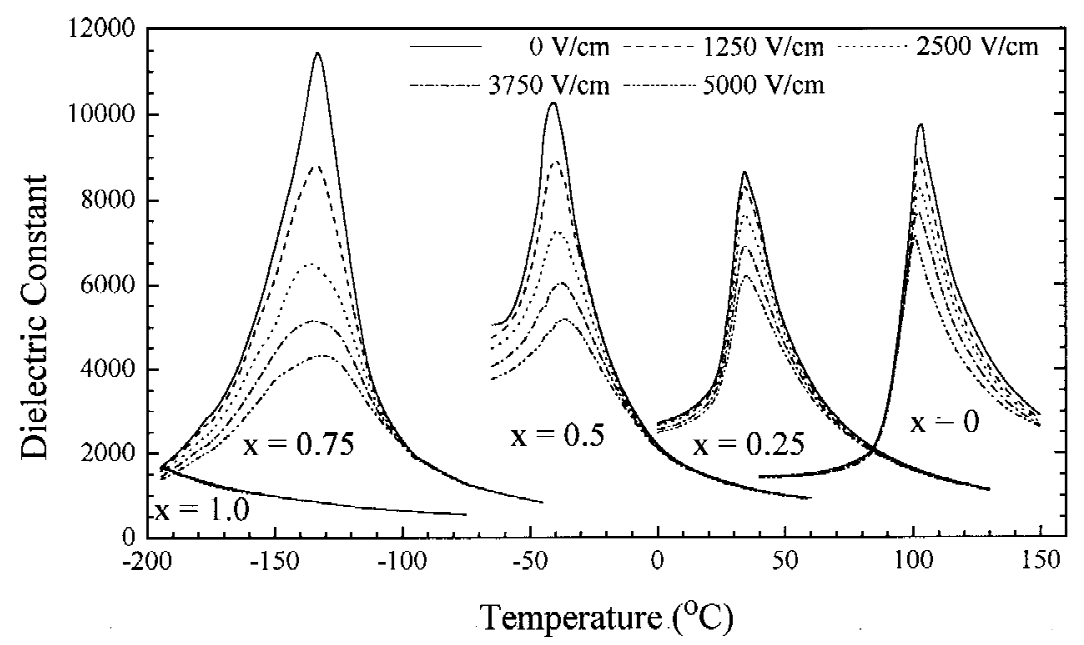

Fig. 3. Temperature dependence of dielectric constant as a function of dc-biasing field for doped BST system. Measuring frequency is $10 \mathrm{kHz}$.

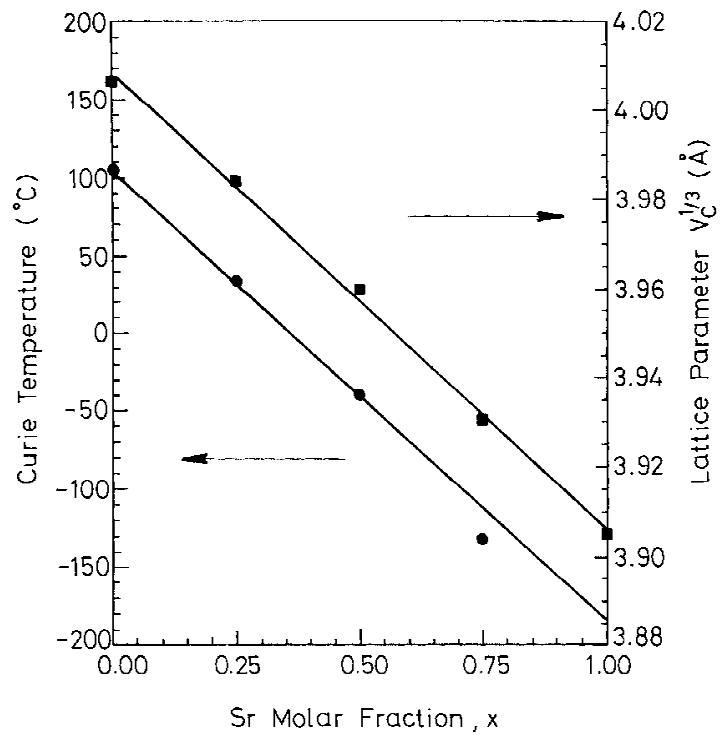

Fig. 4. Lattice parameter $V_{\mathrm{C}}^{1 / 3}$ and $T_{\mathrm{C}}$ of doped BST system.

the second derivative of free energy (Eq. (3)) with respect to polarization:

$$
\frac{4 \pi}{k^{\prime}}=2 A(T-\theta)+12 B P^{2}
$$

In the case of a small field $E$, it is assumed that $k^{\prime} E \approx 4 \pi P$ can be substituted into the second term in Eq. ( $4 a$ ). Thus, a representation of the dielectric constant is obtained as

$$
\frac{4 \pi}{k^{\prime}}=2 A(T-\theta)+\frac{3 B k^{\prime 2} E^{2}}{4 \pi^{2}}
$$

Hence, the dielectric constant varies with dc-biasing $E$. Equation $(4 b)$ reduces to the Curie-Weiss law when the anharmonic term is negligible. The lattice anharmonic interaction of titanium ions is responsible for the field dependence of the dielectric constant of the BST system. Rearranging Eq. (4b), with some approximation, ${ }^{21}$ one can obtain a phenomenological equation that is valid in the paraelectric state:

$\frac{k^{\prime}}{k_{0}^{\prime}}=\frac{1}{\left(1+a k_{0}^{\prime 3} E^{2}\right)^{1 / 3}}$ where $a=12 B /(4 \pi)^{3}$ is the phenomenological coefficient (or anharmonic coefficient), which is derived from the anharmonic term in the Helmholtz free energy. Johnson ${ }^{21}$ has shown that the imaginary part of the relative permittivity of $\mathrm{Ba}_{0.6} \mathrm{Sr}_{0.4} \mathrm{TiO}_{3}$ follows another phenomenological equation:

$$
\frac{k^{\prime \prime}}{k_{0}^{\prime \prime}}=\frac{1}{\left(1+a k_{0}^{\prime 3} E^{2}\right)^{2 / 3}}
$$

where $k_{0}^{\prime \prime}$ and $k^{\prime \prime}$ are the imaginary parts of the relative permittivity under zero bias field and under bias field $E$, respectively. The anharmonic coefficient $a$ is supposed to be an order parameter of the anharmonic interactions. According to Eq. (5) the $k^{\prime} / k_{0}^{\prime}$ ratio decreases as the anharmonic coefficient $a$, zerobias dielectric constant $k_{0}^{\prime}$, and/or applied bias field $E$ increase. Because $k_{0}^{\prime}$ is larger at the Curie peak, the dielectric tunability at $T_{\mathrm{C}}$ is larger than that at $T_{\mathrm{C}}+10^{\circ} \mathrm{C}$ for all compositions, as listed in Table II. From a practical perspective, the domain movement and hysteresis under dc biasing in the ferroelectric state yield nonpredictable dielectric characteristic changes. Thus, although the dielectric tunability at $T_{\mathrm{C}}$ is larger than that at $T_{\mathrm{C}}+10^{\circ} \mathrm{C}$, the suggested working temperature is in the range from $T_{\mathrm{C}}+5^{\circ} \mathrm{C}$ to $T_{\mathrm{C}}+15^{\circ} \mathrm{C}$, where the material resides in the paraelectric state and the dielectric tunability remains appreciable.

Figure 5 exhibits the field dependence of the dielectric constant and loss tangent at $T_{\mathrm{C}}+5^{\circ} \mathrm{C}, T_{\mathrm{C}}+10^{\circ} \mathrm{C}$, and $T_{\mathrm{C}}+15^{\circ} \mathrm{C}$. The solid lines for the dielectric constant are obtained by curve fitting with the phenomenological equation (Eq. (5)). The wellfitted curves, as shown in the upper part of Fig. 5, suggest that the dc field applied up to $5000 \mathrm{~V} / \mathrm{cm}$ is small enough such that neglecting the $P^{6}$ term in Eq. (3) is appropriate. The anharmonic coefficients obtained by curve fitting with Eq. (5) are summarized in Table II. These coefficients increase with temperature and strontium fraction, except for the composition $x=0$. The ionic radii of $\mathrm{Ba}^{2+}$ and $\mathrm{Sr}^{2+}$ are 1.36 and $1.16 \AA$, respectively. ${ }^{9}$ The smaller $\mathrm{Sr}^{2+}$ ion makes the unit cell smaller and the crystal more compact, as indicated in Table I. This results in a shorter distance between the $\mathrm{Ti}^{4+}$ ion and its nonnearest neighbors. That is, the thermal vibration amplitude of the $\mathrm{Ti}^{4+}$ ion is softened more easily by these nonnearest neighbors. This enhances the anharmonic effect, and, consequently, the anharmonic coefficient increases with strontium content for $0.25 \leq x \leq 0.75$. Devonshire's theory assumes a stress-free condition. However, there is internal stress among grains that affect the microscopic value of the dielectric constant. The internal stress decreases with increasing grain size. ${ }^{19}$ As summarized in Table $\mathrm{I}$, the grain size of doped $\mathrm{BaTiO}_{3}$ is the largest, and the internal stress effect should be the smallest. 
Table II. Dielectric Tunability and Anharmonic Coefficients for the 1.0-mol\%-MgO- and 0.05-mol\%-MnO -doped $\mathrm{Ba}_{1-x} \mathrm{Sr}_{x} \mathrm{TiO}_{3}$ System

\begin{tabular}{|c|c|c|c|c|c|}
\hline \multirow{2}{*}{$\begin{array}{l}\text { Strontium molar } \\
\text { fraction }\end{array}$} & \multirow{2}{*}{$\begin{array}{l}\text { Dielectric tunability }{ }^{\dagger} \\
\text { at } T_{\mathrm{C}}(\%)\end{array}$} & \multirow{2}{*}{$\begin{array}{l}\text { Dielectric tunability } \\
\text { at } T_{\mathrm{C}}+10^{\circ} \mathrm{C}(\%)\end{array}$} & \multicolumn{3}{|c|}{$\begin{array}{c}\text { Anharmonic coefficient, } a \\
\left(10^{-19} \mathrm{~cm}^{2} \cdot \mathrm{V}^{-2}\right)\end{array}$} \\
\hline & & & $\overline{T_{\mathrm{C}}+5^{\circ} \mathrm{C}}$ & $T_{\mathrm{C}}+10^{\circ} \mathrm{C}$ & $T_{\mathrm{C}}+15^{\circ} \mathrm{C}$ \\
\hline$x=0$ & 34 & 28 & 1.81 & 2.45 & 3.31 \\
\hline$x=0.25$ & 30 & 17 & 1.09 & 1.26 & 1.85 \\
\hline$x=0.5$ & 51 & 26 & 2.16 & 2.34 & 2.45 \\
\hline$x=0.75$ & 63 & 36 & 4.63 & 5.90 & 6.65 \\
\hline
\end{tabular}

Dielectric tunability is defined by the percentage change of the dielectric constant under $5000 \mathrm{~V} / \mathrm{cm}$ dc-biasing field.
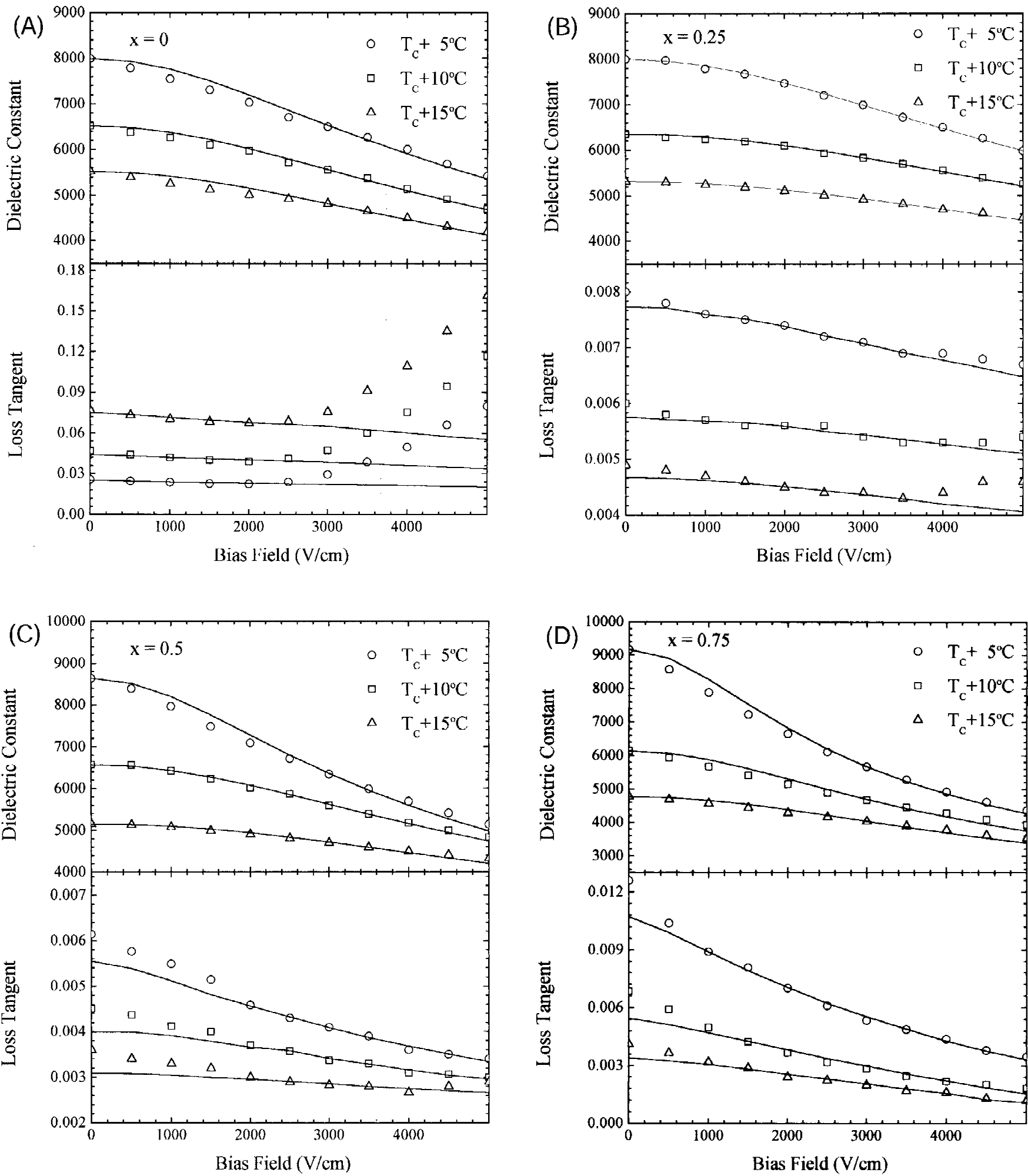

Fig. 5. $10 \mathrm{kHz}$ dielectric constant and loss tangent as a function of dc biasing field for doped BST: (A) $x=0$, (B) $x=0.25$, (C) $x=0.5$, and (D) $x=0.75$. Circle, triangle, and square represent the data at $T_{\mathrm{C}}+5^{\circ} \mathrm{C}, T_{\mathrm{C}}+10^{\circ} \mathrm{C}$, and $T_{\mathrm{C}}+15^{\circ} \mathrm{C}$ respectively. Solid lines are the theoretical curve fitted by Devonshire's theory. 


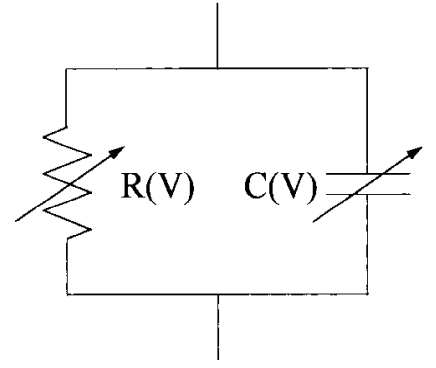

Fig. 6. Circuit to represent the tunable capacitor under dc biasing.

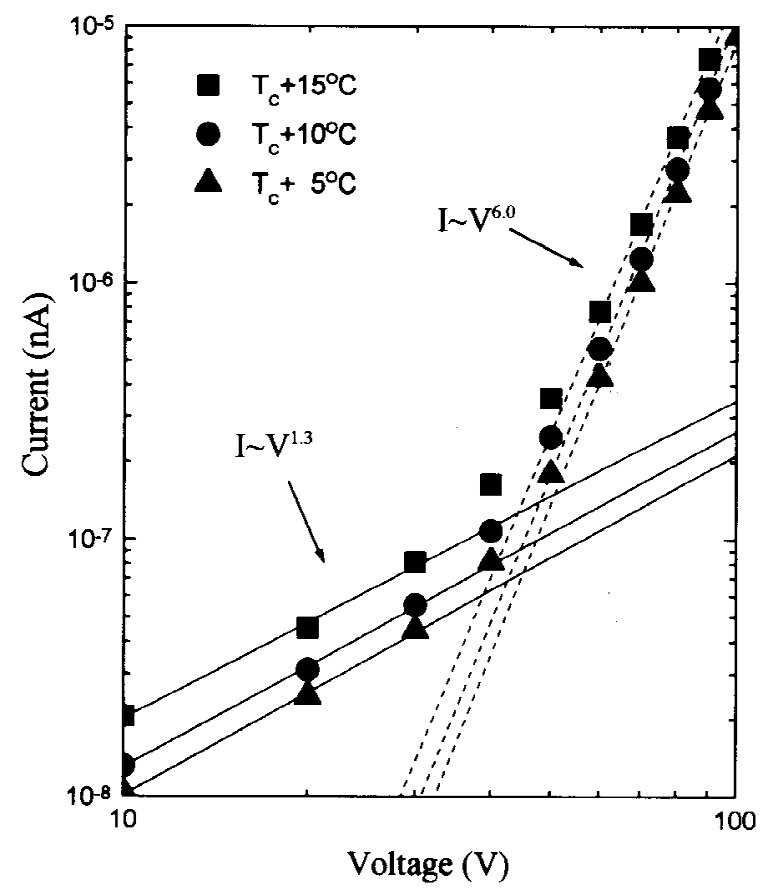

Fig. 7. $I-V$ characteristics for doped $\mathrm{BaTiO}_{3}$ at $T_{\mathrm{C}}+5^{\circ} \mathrm{C}, T_{\mathrm{C}}+10^{\circ} \mathrm{C}$, and $T_{\mathrm{C}}+15^{\circ} \mathrm{C}$.

With little influence of internal stress, the anharmonic interactions and the anharmonic coefficients are enhanced. In Fig. 3, the change of the dielectric constant of $\mathrm{BaTiO}_{3}$ is obvious at the paraelectric state, as compared to that at the ferroelectric state. For $0.25 \leq x \leq 0.75$ in Fig. 3, this change becomes obvious at both ferroelectric and paraelectric states. This change also contributes to the internal stress effect, which broadens the phase transition and, hence, "dilutes" the contribution of the anharmonic coefficient.

The dielectric losses also are reduced by the applied dc field for all the compositions, as shown in the lower part of Fig. 5. On the basis of Eqs. (5) and (6), one obtains the equation for the dielectric loss $\tan \delta_{\mathrm{C}}$.

$$
\tan \delta_{\mathrm{C}}=\frac{k^{\prime \prime}}{k^{\prime}}=\frac{\tan \delta_{\mathrm{C} 0}}{\left(1+a k_{0}^{\prime 3} E^{2}\right)^{1 / 3}}
$$

where $\tan \delta_{\mathrm{C} 0}$ is the dielectric loss under zero bias (i.e., $k_{0}^{\prime \prime} / k_{0}^{\prime}$ ). The solid lines for the field dependence of dielectric loss shown in Fig. 5 are the theoretical curves calculated by Eq. (7) with the anharmonic coefficients listed in Table II. The measured dielectric loss is in good agreement with the theoretical prediction, except in the high-field region of the composition for $x=0$. Doped $\mathrm{BaTiO}_{3}$ (i.e., $x=0$ ) is the most lossy material among the compositions studied. The dielectric loss for $x=0$ in the high-field region $(E>2500 \mathrm{~V} / \mathrm{cm})$ increases with the bias field. There are other dielectric loss mechanisms that result in

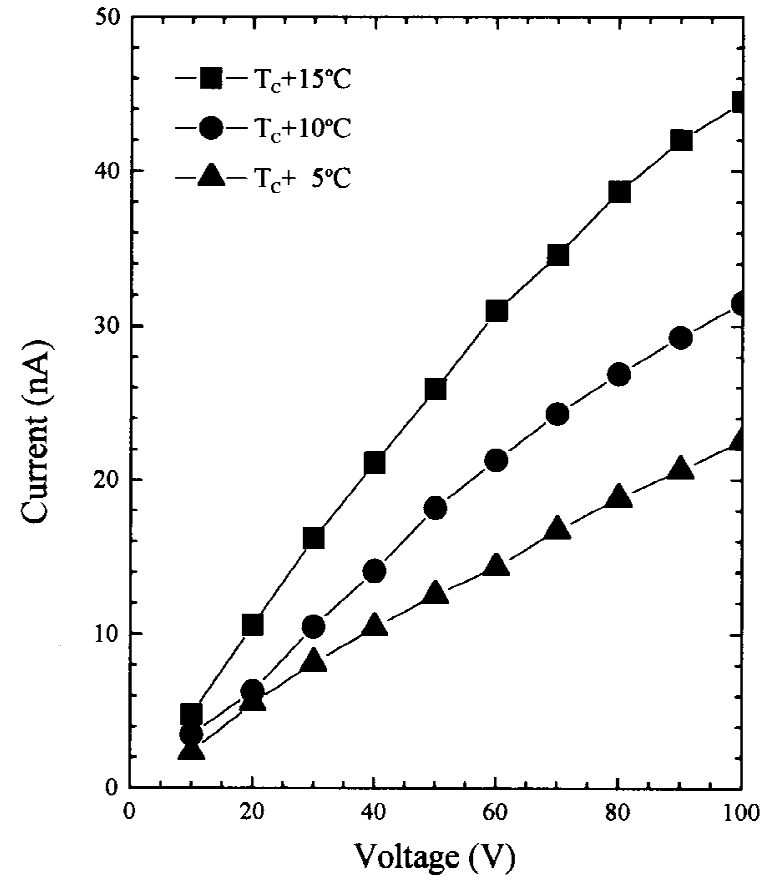

Fig. 8. $I-V$ characteristics for doped $\mathrm{Ba}_{0.75} \mathrm{Sr}_{0.25} \mathrm{TiO}_{3}$ at $T_{\mathrm{C}}+5^{\circ} \mathrm{C}$, $T_{\mathrm{C}}+10^{\circ} \mathrm{C}$, and $T_{\mathrm{C}}+15^{\circ} \mathrm{C}$.

increasing dielectric loss with dc field. In a tunable capacitor, one loss term is attributed to leakage current under dc biasing. The leakage current can be represented as a dc resistor in a parallel combination with a capacitor for dielectric features. As shown in Fig. 6, the dc resistor and the capacitor are voltage variable for a tunable capacitor. Thus, the resistance and the capacitance obey the following relations:

$$
\begin{aligned}
& R(V)=\frac{V}{I(V)} \\
& C(V)=C_{0}\left(k^{\prime}-j k^{\prime \prime}\right)
\end{aligned}
$$

where $I(V)$ is the $I-V$ characteristic of the doped BST system, $C_{0}$ the geometric capacitance, and $k^{\prime}$ and $k^{\prime \prime}$ the real and imaginary parts of the relative permittivity, respectively. Equations (8) and (9) show that the dielectric features of the tunable capacitor are related to the $I-V$ characteristics and to the field dependence of relative permittivity (referring to Eqs. (6) and (7)). Therefore, at some specific measuring frequency $\omega$, the measured dielectric loss tan $\delta$ can be expressed as

$$
\tan \delta=\tan \delta_{\mathrm{C}}+\tan \delta_{\mathrm{R}}=\tan \delta_{\mathrm{C}}+\frac{1}{k^{\prime} \omega R C_{0}}
$$

where $\tan \delta_{\mathrm{C}}$ is the dielectric loss of the voltage-variable capacitor as described in Eq. (7) and $\tan \delta_{\mathrm{R}}$ the dielectric loss that corresponds to the leakage current under dc biasing. $\tan \delta_{R}$ is inversely proportional to the dc resistance.

The $I-V$ characteristics for $x=0$ at $T_{\mathrm{C}}+5^{\circ} \mathrm{C}, T_{\mathrm{C}}+10^{\circ} \mathrm{C}$, and $T_{\mathrm{C}}+15^{\circ} \mathrm{C}$ are shown in Fig. 7. The leakage current increases with increasing applied voltage and temperature. In the low-field region $(V \leq 40 \mathrm{~V})$, the leakage current follows as a powers form of $I \propto V^{1.3}$. However, in the high-field region $(V \geq 50 \mathrm{~V}), I \propto V^{6}$. According to the space charge limited currents (SCLC) theory for polycrystalline insulators, ${ }^{22}$ strongly defected surfaces of the crystalline grains provide a source of deep trap states, where the trapped charge carriers are excited by the applied voltage and thermal energy. Below an onset voltage, an ohmic current flows. For higher voltage, the current transfers to a rapidly increasing behavior of the form $I$ $\propto V^{\alpha}$, where $\alpha$ is in the range 1.2-2.0. ${ }^{23}$ As the voltage reaches 
a traps-filled limited voltage, the current increases more rapidly, and the $I \propto V$ characteristics depend on the trap distributions of the form $I \propto V^{\mathrm{m}}(m=2,4,6, \ldots)$ or $I \propto e^{\alpha V}$. Figure 7 shows that the ohmic region is not present when the applied voltage is $>10 \mathrm{~V}$. The traps-filled limited voltage is $\sim 50 \mathrm{~V}$. The $I-V$ characteristics in the high-field region follow the form $I \propto$ $V^{6}$ and imply that the traps are exponentially distributed in the energy gap. The dc resistance $R(V)$ for $x=0$ calculated from Eq. (8) is a function of voltage and decreases rapidly with it. The $I-V$ characteristics for $x=0.25$ are shown in Fig. 8. Over the applied voltage range, the $I-V$ characteristics are all in the ohmic region. At $T_{\mathrm{C}}+5^{\circ} \mathrm{C}$, the leakage current for $x=0.25$ $(\sim 22.53 \mathrm{nA}$ at $100 \mathrm{~V})$ is much lower than that for composition $x=0(\sim 9170 \mathrm{nA}$ at $100 \mathrm{~V})$. The dc resistivities for $x=0.25$ are $51.3,37.0$, and $25.2 \mathrm{G} \Omega \cdot \mathrm{cm}$ at $T_{\mathrm{C}}+5^{\circ} \mathrm{C}, T_{\mathrm{C}}+10^{\circ} \mathrm{C}$, and $T_{\mathrm{C}}+15^{\circ} \mathrm{C}$, respectively. With these values and Eq. (10), the dielectric loss corresponding to the dc-resistor $\tan \delta_{\mathrm{R}}$ is evaluated be 0.0005 for $T_{\mathrm{C}}+5^{\circ} \mathrm{C}, 0.0008$ for $T_{\mathrm{C}}+10^{\circ} \mathrm{C}$, and 0.0014 for $T_{\mathrm{C}}+15^{\circ} \mathrm{C}$. These values are much lower than the measured dielectric loss shown in Fig. 5(b). Therefore, the dielectric loss for $x=0.25$ is dominated by the loss from the voltage-variable capacitor, i.e., $\tan \delta_{\mathrm{C}}$ in Eq. (10), and, thus, the theoretical curves, as described by Eq. (7), fit well. But, for the case of $x=0$, the two loss mechanisms described in Eq. (10) compete with each other. Figure 5(a), in the low-field region, shows that the specimen is of such good insulation that the dielectric loss from the voltage-variable capacitor dominates. As the voltage increases, the rapid decrease in resistance yields a rapid increase in the dielectric loss tan $\delta_{R}$ from the dc resistance. Thus, $\tan \delta_{R}$ is important in the dielectric loss. This results in an increase of dielectric loss with dc bias in the high-field range for $x=0$, as shown in Fig. 5(a).

The grain-growth inhibitor $\mathrm{MgO}$ also acts as an acceptor in $\mathrm{BaTiO}_{3}$ ceramics. It is reported that the $\mathrm{Mg}^{2+}$ ion substitutes in the $\mathrm{Ti}^{4+}$ site up to $0.25 \mathrm{~mol} \% .^{24,25}$ As mentioned previously, it is more difficult to dissolve $\mathrm{MgO}$ in strontium-rich samples than in barium-rich samples because of the more refractory nature of strontium-rich samples. Sample grain size decreases with an increase of strontium content as listed in Table I. In other words, $\mathrm{MgO}$ is more effective as an acceptor in a bariumrich sample, which yields more oxygen vacancies and more traps. It is argued that the deviation of the dielectric loss from the theoretical prediction for the composition with $x=0$ (shown in Fig. 5(a)) is attributed to the increase of trap states.

\section{Conclusions}

(1) $\mathrm{Ba}_{1-x} \mathrm{Sr}_{x} \mathrm{TiO}_{3}$ doped with $1.0 \mathrm{~mol} \% \mathrm{MgO}$ and 0.05 mol\% $\mathrm{MnO}_{2}$ has been fabricated by a rate-controlled sintering profile. $\mathrm{MgO}$ was more effective to inhibit the grain growth for strontium-rich composition. The grain size of samples decreases from $14 \mu \mathrm{m}$ for doped $\mathrm{BaTiO}_{3}$ (i.e., $x=0$ ) to $2 \mu \mathrm{m}$ for doped $\mathrm{SrTiO}_{3}$ (i.e., $x=1$ ).

(2) A linear relation, $T_{\mathrm{C}}\left({ }^{\circ} \mathrm{C}\right)=104-288 x$, exists between the Curie temperature and strontium fraction $x$. However, the reported $T_{\mathrm{C}}$ value of $\mathrm{SrTiO}_{3}$ (i.e., the composition at $x=1$ ) is lower than that predicted by this empirical equation. It is believed that the deviation is due to the quantum fluctuation at low temperatures.

(3) For the application of the tunable capacitor, it is suggested that the working temperature is $5^{\circ}-15^{\circ} \mathrm{C}$ above $T_{\mathrm{C}}$ to prevent the instability of the dielectric behavior caused by the phase transition at $\sim T_{\mathrm{C}}$.

(4) The phenomenological equations (Eqs. (5) and (6)) derived on the basis of Devonshire's theory explain the field dependence of the dielectric properties for the paraelectric $\mathrm{Ba}_{1-x} \mathrm{Sr}_{x} \mathrm{TiO}_{3}$ system.
(5) Both the anharmonic coefficient and the dielectric tunability increase as more strontium is presented. It is believed that the smaller $\mathrm{Sr}^{2+}$ ion results in shorter $\mathrm{Ti}^{4+}$ to nonnearest neighbor distance, which enhances the anharmonic effect.

(6) The dielectric loss as a function of dc biasing in the paraelectric state suggests that the dielectric loss comprises two loss mechanisms, which can be represented as a two-component circuit, as depicted in Fig. 6. The loss from the voltagevariable capacitor $\tan \delta_{\mathrm{C}}$ is important in the low-loss materials $(x=0.25,0.5$, and 0.75$)$ of which the dc resistance is large. Loss resulting from the dc resistor, $\tan \delta_{\mathrm{R}}$, dominates when the biasing field is $>2500 \mathrm{~V} / \mathrm{cm}$ for $x=0$.

\section{References}

${ }^{1}$ U. Syamaprasad, R. K. Galgali, and B. C. Mohanty, "Dielectric Properties of the $\mathrm{Ba}_{1-x} \mathrm{Sr}_{x} \mathrm{TiO}_{3}$ System,'” Mater. Lett., 7 [5-6] 197-200 (1988).

${ }^{2}$ B. S. Chiou, S. T. Li, J. G. Duh, and P. H. Chang, "Equivalent Circuit Model in Grain-Boundary Barrier Layer Capacitors,' J. Am. Ceram. Soc., 72 [10] 1967-75 (1989).

${ }^{3}$ K. K. Deb, M. D. Hill, and J. F. Kelly, "Pyroelectric Characteristics of Modified Barium Titanate Ceramics," J. Mater. Res., 7 [12] 3296-305 (1992).

${ }^{4}$ S. L. Swartz and A. S. Bhalla, "Dielectric Properties of $\mathrm{SrTiO}_{3}$ GlassCeramics,' Ferroelectrics, 87, 141-54 (1988).

${ }^{5}$ R. Viana, P. Lunkenheimer, J. Hemberger, R. Böhmer, and A. Loidl, “'Dielectric Spectroscopy in $\mathrm{SrTiO}_{3}$,' Phys. Rev. B: Condens. Matter, 50 [1] 601604 (1994).

${ }^{6}$ B. S. Chiou, C. M. Koh, and J. G. Duh, "'The Influence of Firing Profile and Additives on the PTCR Effect and Microstructure of $\mathrm{BaTiO}_{3}$ Ceramics,' $J$. Mater. Sci., 22, 3893-900 (1987).

${ }^{7}$ B. S. Chiou and S. T. Lin, "Dielectric Characteristics of LiF-Doped $\mathrm{BaTiO}_{3}$ with $\mathrm{Nb}_{2} \mathrm{O}_{5}$ and $\mathrm{Dy}_{2} \mathrm{O}_{3}$ Addition,'” Mater. Chem. Phys., 20, 431-50 (1988).

${ }^{8}$ F. Batllo, E. Duverger, J. C. Jules, J. C. Niepce, B. Jannot, and M. Maglione, "Dielectric and EPR Studies on Mn-Doped Barium Titanate,', Ferroelectrics, 109, 113-18 (1990).

${ }^{9}$ S. B. Herner, F. A. Selmi, V. V. Varadan, and V. K. Varadan, "The Effect of Various Dopants on the Dielectric Properties of Barium Strontium Titanate," Mater. Lett., 15, 317-24 (1993).

${ }^{10}$ R. W. Babbitt, T. E. Koscica, and W. C. Drach, "Planar Microwave Electro-optic Phase Shifters,' Microwave J., [June] 63-79 (1992).

${ }^{11}$ F. Selmi, R. Hughes, V. K. Varadan, and V. V. Varadan, “Tunable Ceramic Phase Shifters and Their Applications," Proc. Soc. Photo-Opt. Instrum. Eng., 1916, 180-88 (1993).

${ }^{12}$ D. Galt, J. C. Price, J. A. Beall, and R. H. Ono, "Characterization of a Tunable Thin-Film Microwave $\mathrm{YBa}_{2} \mathrm{Cu}_{3} \mathrm{O}_{7-x} / \mathrm{SrTiO}_{3}$ Coplanar Capacitor,' Appl. Phys. Lett., 63 [29] 3078-80 (1993).

${ }^{13}$ A. Outzourhit, U. Trefny, T. Kito, and B. Yarar, "Tunability of the Dielectric Constant of $\mathrm{Ba}_{0.1} \mathrm{Sr}_{0.9} \mathrm{TiO}_{3}$ Ceramics in the Paraelectric State,' J. Mater. Res., 10 [6] 1411-17 (1995).

${ }^{14}$ A. Outzourhit, J. U. Trefny, T. Kito, B. Yarar, A. Naziripour, and A. M. Hermann, "Fabrication and Characterization of $\mathrm{Ba}_{1-x} \mathrm{Sr}_{x} \mathrm{TiO}_{3}$ Tunable ThinFilm Capacitors," Thin Solid Films, 259, 218-24 (1995).

${ }^{15} \mathrm{H}$. U. Anderson, "Influence of Ba/Ti Ratio on the Initial Sintering Kinetics of $\mathrm{BaTiO}_{3}$,' J. Am. Ceram. Soc., 56 [11] 605-606 (1973).

${ }^{16}$ T. T. Fang and H. L. Hsieh, "The Effects of Pore Morphology and Microstructure on the Dielectric Properties of High-Purity $\mathrm{BaTiO}_{3}$,' Chin. J. Mater. Sci., 21 [2] 127-35 (1989).

${ }^{17}$ B. W. Lee and K. H. Auh, "Effects of Grain Size and Mechanical Process on the Dielectric Properties of $\mathrm{BaTiO}_{3}$,' J. Mater. Res., 10 [6] 1418-23 (1995).

${ }^{18}$ K. Kinoshita and A. Yamaji, "Grain-Size Effects on Dielectric Properties in Barium Titanate Ceramics,'” J. Appl. Phys., 47 [1] 371-73 (1976).

${ }^{19}$ H. T. Martirena and J. C. Burfoot, "Grain-Size Effects on Properties of Some Ferroelectric Ceramics,', J. Phys. C: Solid State Phys., 7, 3182-92 (1974).

${ }^{20}$ G. Arlt, D. Hennings, and G. de With, "Dielectric Properties of FineGrained Barium Titanate Ceramics,'” J. Appl. Phys., 58 [4] 1619-25 (1985).

${ }^{21}$ K. M. Johnson, "Variation of Dielectric Constant with Voltage in Ferroelectrics and Its Application to Parametric Devices," J. Appl. Phys., 33 [9] 2826-31 (1962)

${ }^{22} \mathrm{H}$. Nemoto and I. Oda, "Direct Examination of PTC Action of SingleGrain Boundaries in Semiconducting $\mathrm{BaTiO}_{3}$ Ceramics,' J. Am. Ceram. Soc., 63 [7-8] 398-401 (1980).

${ }^{23} \mathrm{~W}$. Osak and K. Tkacz, "Investigation of $I-V$ Characteristics in Polycrystalline BaTiO ${ }_{3}$,' J. Phys. D: Appl. Phys., 22, 1746-50 (1989).

${ }^{24} \mathrm{G}$. V. Lewis and C. R. A. Catlow, "PTCR Effect in $\mathrm{BaTiO}_{3}$," J. Am. Ceram. Soc., 68 [10] 555-58 (1985).

${ }^{25}$ C. J. Peng and H. Y. Lu, "Compensation Effect in Semiconducting Barium Titanate,'” J. Am. Ceram. Soc., 71 [1] C-44-C-46 (1988). 